\title{
Constraining the star formation history with Fermi-LAT observations of the gamma-ray opacity of the universe
}

\author{
Ashlynn Le Ray*† \\ School of Physics \\ University of the Witwatersrand, Johannesburg \\ South Africa \\ E-mail: 755015estudents.wits.ac.za \\ Andrew Chen \\ School of Physics \\ University of the Witwatersrand, Johannesburg \\ South Africa \\ E-mail: andrew.chen@wits.ac.za
}

\begin{abstract}
The star formation history (SFH) of the Universe is of fundamental importance to cosmology, not only to galactic formation itself but also for ongoing efforts to determine cosmological parameters and matter content of the Universe [1]. Measurements of the extragalactic background light (EBL) as a function of redshift can constrain models of the SFH, including the initial mass function(IMF) and dust extinction [1]. The gamma-ray spectra of AGN allows us to study the extragalactic background light (EBL) through $\gamma-\gamma$ absorption of high-energy photons. In this work, we will produce constraints on models of SFH using a sample of AGN observed by the FermiLAT instrument by investigating the imprint of the EBL on the observed spectra of high-redshift Fermi-LAT AGN above $\sim 10 \mathrm{GeV}$.
\end{abstract}

High Energy Astrophysics in Southern Africa - HEASA2018

1-3 August, 2018

Parys, Free State, South Africa

\footnotetext{
* Speaker.

${ }^{\dagger}$ A footnote may follow.
} 


\section{Introduction}

The extragalactic background light (EBL) consists of photons emitted at all epochs by stars, dust and active galactic nuclei(AGN), providing information about the evolution of galaxies from very early times up to the present [2]. A vast majority of the EBL energy is found in two wavelength ranges, namely, optical and near-infrared [2]. The optical light consists of direct light from stars, and the infrared light is produced by thermal radiation from dust. At longer wavelengths, the background spectrum is dominated by the cosmic microwave background. A schematic showing the EBL intensity distribution as a function of wavelength/frequency of the EBL photon is shown in Figure 1. The cosmic optical background (COB) is primarily due to direct radiation from stars and galaxies, whereas the absorption and re-radiation of light by dust particles is responsible for the cosmic infrared background CIB [3]. The EBL tracks galactic and stellar evolution, and varies with redshift[9].

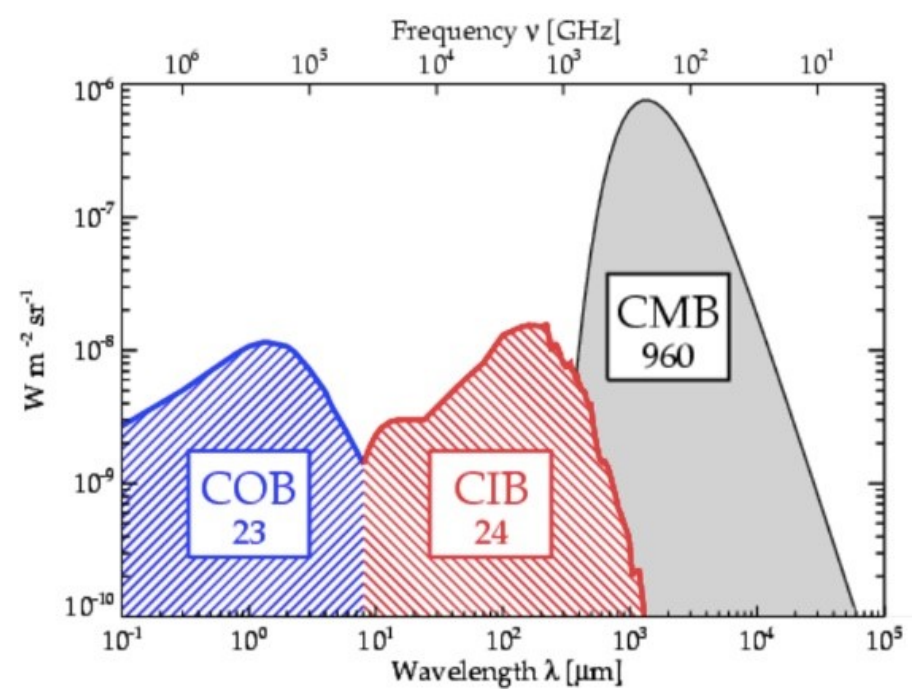

Figure 1: This figure depicts the EBL intensity as a function of wavelength/frequency. Figure adopted from [4].

The EBL designates just one segment of the diffuse photon field present in the universe: ultraviolet, radio, X-ray and $\gamma$-ray backgrounds are also present, as well as the cosmic microwave background (CMB) [3]. An indirect means of probing the diffuse radiation fields is through $\gamma-\gamma$ absorption of high-energy gamma rays. In this process, a gamma-ray photon from a distant astrophysical source of energy $E_{\gamma}$ and an extragalactic background light (EBL) photon of energy $E_{E B L}$ annihilate and create an electron-positron pair [5].

\section{Blazars}

An active galactic nucleus(AGN) has a disk of material surrounding the central supermassive black hole. If the AGN is also ejecting jets, and the jets happen to point toward us, it is considered a blazar. Blazars are divided into two categories: BL Lacertae(BL Lac) and flat spectrum Radio Quasar (FSRQ)[10]. 


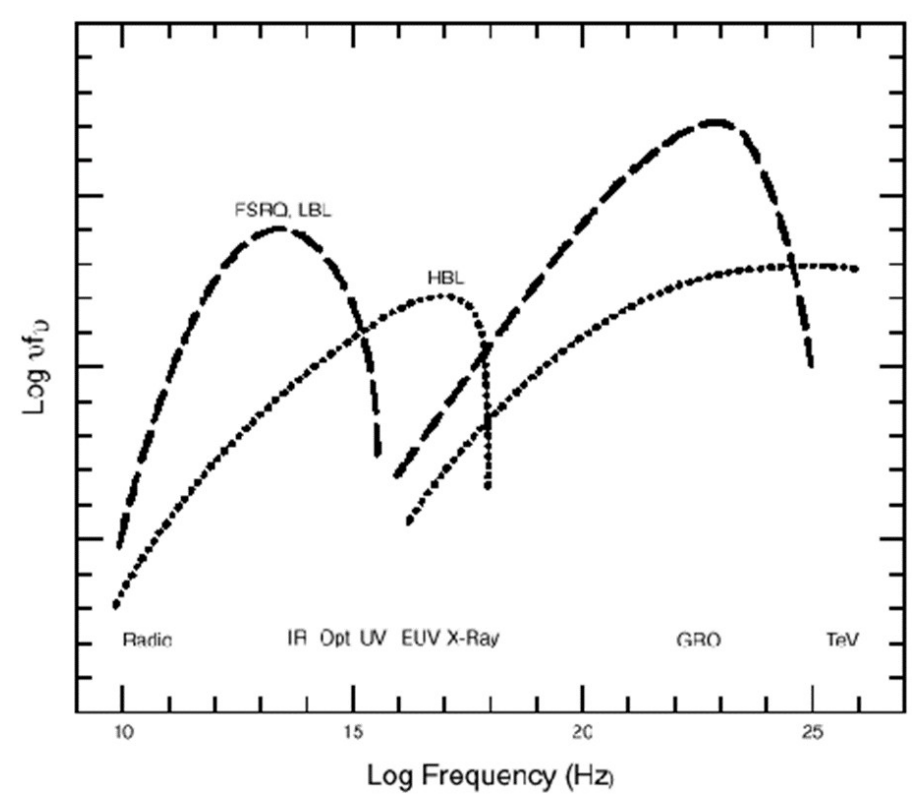

Figure 2: Spectral Energy Distribution for three kinds of blazars. Credit: Padovani, P., and Giommi, P. MNRAS, 277, 1477 (1995).

One difference between the two subclasses (BL Lacertae [BL Lac] objects and flat-spectrum radio sources [FSRQs]) is the intrinsic gamma-ray spectrum on average of each type of source. The intrinsic gamma-ray spectrum of FSRQs is curved in the Fermi region so if we were to study these objects, our task would be to determine how much curvature is due to the intrinsic $\gamma$-ray spectrum and how much curvature is due to the EBL. However, the intrinsic gamma-ray spectrum of many BL Lacs is flat in the Fermi region, so the curvature observed in the spectrum is due to $\gamma-\gamma$ attenuation (See Figure 2).

\section{Computation of the Extragalctic Background Light (EBL)}

High-energy gamma-rays can interact with EBL photons in electron-positron pair production interactions ([11]; [12]; [13]). By effectively removing these gamma-rays from view, this process alters the observed spectra of extragalactic high-energy sources, and increasingly occludes those at higher redshifts. The rapid development of ground-based gamma-ray astronomy in the past 20 years has led to a number of attempts (i.e. [20], [21], [22], [23]) to constrain the EBL based on modification to gamma-ray spectra. A method that can provide a measurement of the EBL that is independent of direct observation. Understanding how the EBL is produced and how its spectral energy distribution (SED) evolves with redshift requires an understanding of the sources responsible for its production. Calculations of the EBL fall into four general categories: i) forward evolution, which begin with initial stellar populations, an assumed cosmic star formation rate, and follow galaxy evolution forward in time. Semi-analytic models (SAMs) are examples of forward evolution models[1]; ii) backwards evolution of the well-constrained present-day galaxy emissivity according to some prescription; iii) evolution of galaxy properties that are inferred over some range in wavelength; iv) direct observation of evolution in galaxy properties over the redshifts providing the major contribution to the background light[8]. 


\section{EBL calculation and model predictions}

The temporal evolution of the specific luminosity, $L_{v}(t)$ (in units of erg $s^{-1} \mathrm{~Hz}^{-1}$ per unit mass of stars formed) is determined by the choices of IMF and the initial stellar metallicity( the spectral energy distribution of a stellar population as a function of age for a Salpeter IMF between 0.1 and $100 M_{\odot}$ is shown below(See figure 3 )). The comoving emissivity per unit volume is obtained by integrating the specific luminosity density and the star formation rate per comoving unit volume over redshift, for a given cosmology.

$$
\varepsilon_{v}(z)=\int_{z}^{z_{m}} L_{v}\left(t(z)-t\left(z^{\prime}\right) \dot{\rho}_{*}\left(z^{\prime}\right)\left|\frac{d t^{\prime}}{d z^{\prime}}\right| d z^{\prime}\right.
$$

where $\dot{\rho}_{*}\left(z^{\prime}\right)$ is the star formation rate per comoving unit volume. The star formation rate is assumed to have begun at some finite epoch $z_{m}=z\left(t_{m}\right)$.

A second integration over redshift for the evolution of the emissivity, yields the energy density, when multiplied by $c / 4 \pi$, yields the power spectrum of the EBL.

$$
P_{v}(z)=v I_{v}(z)=\int_{z}^{z_{m}} \varepsilon_{v^{\prime}}\left(z^{\prime}\right)\left|\frac{d t^{\prime}}{d z^{\prime}}\right| d z^{\prime}
$$

with $v^{\prime}=v\left(1+z^{\prime}\right)(1+z)$.

The photon number density

$$
n(\varepsilon, z)=\frac{P_{v}(z)}{E}
$$

where $E=h v$ is the energy of a photon, h is Planck's constant.

The infrared emission is modelled as the sum of three modified blackbody spectra(see equation 5.1). The temperatures of these components characterize warm and cold dust in galaxies and a Polycyclic aromatic hydrocarbon(PAH) component, which is assumed to emit like a Blackbody. The number density is obtained by dividing the comoving power spectrum of the EBL by the energy of a photon.The optical depth is obtained by integrating the number density(see equation 4.3), pair production cross-section and the angle between interacting photons over suitable boundary conditions, see equation (6.1). New optical depth data is used to constrain the parameter values obtained by the gamma-ray opacity models developed by [7], to the measured values of the gammaray opacity obtained by the Fermi analysis team.

\section{Comparison of models to data}

The re-emission is calculated as the sum of three modified Planck spectra

$$
L_{\lambda}^{d}\left(L_{b o l}\right)=\sum_{i=1}^{\infty} c_{i}\left(L_{b o l}\right) Q_{\lambda} B_{\lambda}\left(T_{i}\right)
$$

where $Q_{\lambda} \propto \lambda^{-1}, L_{b o l}=L_{b o l}(\tau)$ and here $\tau$ is the age of the stellar population. The cold and warm 

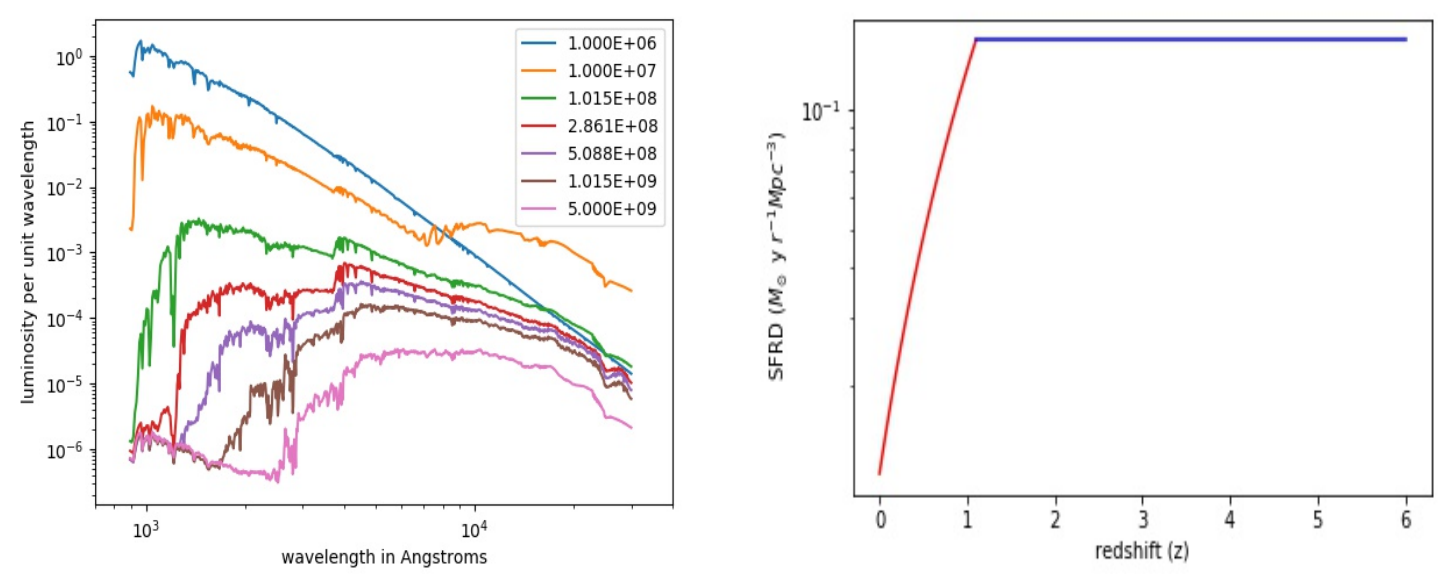

Figure 3: Left panel: Spectral energy distribution (SEDs) of a coeval stellar population as a function of age $\tau$. The specific luminosity of the evolving stellar population is normalized to one solar mass. This figure depicts the SEDs for ages $\tau, 0.0010,0.01,0.1,0.3,0.5,1$ and 5 Gyrs. Right panel: Comoving star formation rate density as a function of redshift for the following parameters $, \alpha=3.4, \beta=0, z_{\text {peak }}=1.1, \dot{\rho}_{*}\left(z_{\text {peak }}\right)=0.15 M_{\odot} \mathrm{y} r^{-1} M p c^{-3}$.
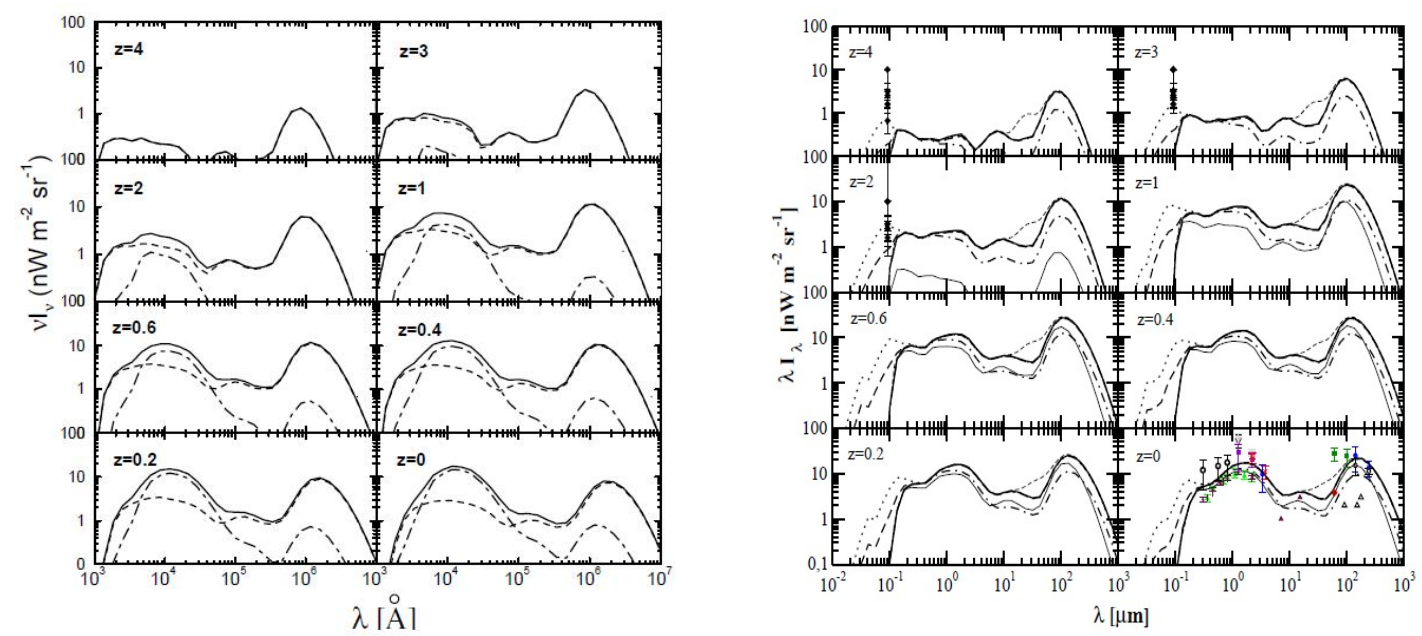

Figure 4: Left panel: The evolving spectrum of the extragalactic background light as a function of wavelength (in Angstroms).The contribution of massive stars are depicted by dashed lines and the contribution of low mass stars are denoted by the dot-dashed line in the left panel. Figure adopted from [8]. Right panel: Comoving EBL at various redshifts. The thick solid line depicts the "Bestfit" model, the thin dashed line depicts the "Warm-Dust" model; "Low-IR" model, dot-dashed line; "Low-SFR" model is depicted by the thin solid line. Figure adopted from [9]

dust, and the contribution due to PAH molecules are characterized by the following components, $\left(c_{1}, T_{1}\right),\left(c_{2}, T_{2}\right)$ and $\left(c_{3}, T_{3}\right)$, respectively. The best-fit values for the parameters are obtained from sample galaxies detected with Infrared Astronomical Satellite (IRAS) at $12 \mu \mathrm{m}, 25 \mu \mathrm{m}, 60 \mu \mathrm{m}$, 
and $100 \mu \mathrm{m}$. The global star formation rate (SFR) $\dot{\rho}_{*}(z)$ consists of two components[9].

$$
\dot{\rho}_{*}(z)=\dot{\rho}_{*}^{O P T}(z)+\dot{\rho}_{*}^{U L I G}(z)
$$

The first component accounts for the stars detected in the optical region, the other component accounts for the stars hidden by dust, which can only be detected in the infrared region. Each component of the SFR can be approximated with a simple broken power law [9]:

$$
\dot{\rho}_{*}(z) \propto(1+z)^{\alpha}
$$

with $\alpha=\alpha_{m}>0$ for $z \leq z_{\text {peak }}$ and $\alpha=\beta_{m}<0$ for $z>z_{\text {peak }}$. For model parameters, see table 1. in [10].

\section{Gamma-ray attenuation}

The optical depth for $\gamma \gamma$ absorption for a source, originating at redshift $z \sim 2$, and at an observed energy $E_{\gamma}$ is easily calculated from[8]

$$
\tau_{\gamma \gamma}\left(E_{\gamma}, z\right)=\int_{0}^{z} \frac{d l}{d z^{\prime}} d z^{\prime} \int_{0}^{2} d \mu \frac{\mu}{2} \int_{\varepsilon_{\min }}^{\infty} d \varepsilon \sigma_{\gamma \gamma}\left(E_{\gamma}, \varepsilon, \mu, z\right) n(\varepsilon, z)
$$

with the cosmological line element $\frac{d l}{d z^{\prime}}, \mu=\cos \theta$ is the angle between interacting photons, $n(\varepsilon, z)$ is the number density of the EBL as a function of redshift and EBL photon energy and $\sigma_{\gamma \gamma}$ is the pair-production cross section[9].

\section{Conclusion}

The model which is used to calculate the SFRD is a free fit function aimed at reproducing the emissivities derived from deep surveys [7] (Right panel in Figure 3). It is the most suitable model because it is in good agreement with the observationally determined emissivities determined by [18] and [19].

In our current work, we intend to update [7] models with the [24] updated stellar population synthesis models. We intend to constrain the parameters of the star formation history and investigate its effects on the EBL. Our aim is to obtain more compact constraints on the EBL. Opacity data from Fermi observations will be used to constrain the parameters of our models compare our model predictions to data found by [25]

\section{References}

[1] R. Soebur, C.D Dermer, and J. Finke The stellar contribution to the Extragalactic Background Light and absorption of high-energy gamma-rays., A\&A, 697-492, 2009

[2] A. Franceschini, G. Rodighiero, and M. Vaccari Extragalactic optical-infrared background radiation, its time evolution and the cosmic photon-photon opacity, A\&A, 487, 837-852, 2008

[3] E. Pueschel, The Extragalactic Background Light: Constraints from TeV Blazar Observations., in proceedings of 35th International Cosmic Ray Conference- ICRC2017, PoS ( ICRC2017) 1107 (2017). 
[4] N. Bavouzet, H. Dole, E. Le Floc'h, K. I. Caputi, G. Lagache1, and C. S. Kochanek Estimating the total infrared luminosity of galaxies up to $z \sim 2$ from mid-and far-infrared observations, aap 01 (451) 417

[5] M. Ackerman, M. Ajello, Allafort The imprint of the Extragalactic Background Light in the Gamma-Ray Spectra of Blazars. Science, A\&A (451) 417 [hep-th/2006].

[6] P.A.R. Ade Planck 2013 results. XVI. Cosmological parameters, A\&A(451) 417

[7] T.M Kneiske, K. Mannheim, and D.H. Hartman Implications of Cosmological Gamma-ray Absorption.I. Evolution of the Metagalactic Radiation Field., A\&A 386, 1-11 (2002)

[8] T. Armstrong, K. Mannheim, and D.H. Hartman Updated Fermi-LAT constraints on the extragalactic background light. , 7th Fermi Symposium 2017. Garmisch-Partenkirchen, Germany.,

[9] T.M Kneiske, K. Mannheim, and D.H. Hartman Implications of Cosmological Gamma-ray Absorption.II. Modification of gamma-ray spectra, A\&A 413, 807-815 (2004)

[10] C. Megan Urry and P. Padovani Unified schemes for radio-loud active galactic, Publ.Astron.Soc.Pac., 107:803, 1995. doi: 10.1086/133630

[11] Nikishov, A. I. Absorption of high-energy photons in the Universe, Sov. Phys. JETP 14, 393-394 (1962)

[12] J.V. Jelley High-energy $\gamma$-ray absorption in space by a $3.5 \mathrm{~K}$ microwave field, Phys. Rev. Lett. 16479 , 1966

[13] Gould, R. J. \& Schreder, G. P. Opacity of the Universe to high-energy photons, Phys. Rev. Lett. 16, 252-254 (1966)

[14] M. Orr, F. Krennrich, E. Dwek Strong new constraints on the Extragalactic Background Light in the Near- to Mid-Infrared, 32nd International Cosmic Ray Conference, Beiljing 2011

[15] Daniel Mazin, Martin Raue New limits on the density of the Extragalactic Background Light in the optical to the far infrared from the spectra of all known TeV blazars, astro-ph/0701694v3, 27 June 2007

[16] Albert J Very-High-Energy Gamma Rays from a Distant Quasar: How transparent is the Universe?, Science 27 Jun 2008: Vol. 320, Issue 5884, pp. 1752-1754 DOI: 10.1126/science.1157087

[17] F. Aharonian A low level of Extragalactic Background Light as revealed by $\gamma$-rays from blazars, Nature volume 440, pages 1018-1021 (20 April 2006)

[18] S.J. Lilly The Canada-France Redshift Survey: The Luminosity Density and star formation history of the universe to $z \sim 1$, The Astrophysical Journal, 460: L1-L4, 1996 March 20

[19] Ellis, R. S., Colless, M., Broadhurst, T., Heye, J., \& Glazerbrook, K. 1996, MNRAS, 280, 235 Autofib Redshift Survey - I. Evolution of the galaxy luminosity function, MNRAS, 280, 235

[20] Dwek, E., \& Krennrich, F. Simultaneous constraints on the spectrum of the Extragalactic Background Light and the intrinsic TeV spectra of Markarian 421, Markarian 501, and H1426 + 428, 2005, ApJ, 618,657

[21] Aharonian F. A low level of extragalactic background light as revealed by $\gamma$-rays from blazars, 2006, Nat , 440, 1018

[22] Mazin D. Raue M. New limits on the density of the extragalactic background light in the optical to the far infrared from the spectra of all known TeV blazars, 2007, A\&A, 471, 439

[23] Albert J. Very-High-Energy Gamma Rays from a Distant Quasar: How Transparent Is the Universe? , 2008, Sci , 320, 1752 
[24] Bruzual, G. \& Charlot, S. Stellar population synthesis at the resolution of 2003, 2003, MNRAS, 344, 1000

[25] The Fermi-LAT Collaboration A gamma-ray determination of the Universe's star formation history, 30 November 2018, Science 362, 1031 (2018) DOI: 10.1126/science.aat8123 